\title{
Initial Multicenter Experience With Double Nucleoside Therapy for Human Immunodeficiency Virus Infection During Pregnancy
}

\author{
N.S. Silverman, ${ }^{1,2 *}$ D.H. Watts, ${ }^{3}$ J. Hitti, ${ }^{4}$ D.M. Money, ${ }^{5}$ \\ E. Livingston, ${ }^{6}$ J. Axelrod, ${ }^{6}$ J.M. Ernest, ${ }^{7}$ D. Robbins, ${ }^{1}$ and \\ M.M. DiVito ${ }^{1}$ \\ ${ }^{1}$ Department of Obstetrics and Gynecology, Thomas Jefferson University Hospital, Philadelphia, PA \\ ${ }^{2}$ Department of Obstetrics and Gynecology, Cedars-Sinai Medical Center, Los Angeles, CA \\ ${ }^{3}$ Department of Obstetrics and Gynecology, University of Washington Medical Center, Seattle, WA \\ ${ }^{4}$ Department of Obstetrics and Gynecology, University of Massachusetts Medical Center, \\ Worcester, $M A$ \\ ${ }^{5}$ Department of Obstetrics and Gynecology, University of British Columbia-Oak Tree Clinic, \\ Vancouver, $B C$ \\ ${ }^{6}$ Department of Obstetrics and Gynecology, Duke University Medical Center, Durham, NC \\ ${ }^{7}$ Department of Obstetrics and Gynecology, Wake Forest University School of Medicine, \\ Winston-Salem, $N G$
}

\begin{abstract}
Objective: To study maternal and neonatal effects of combination nucleoside analog therapy administered to human immunodeficiency virus (HIV)-infected pregnant women for maternal indications.

Methods: A multicenter, prospective observational study was undertaken at six perinatal centers in the United States and Canada that supported regional referral programs for the treatment of HIV-infected pregnant women. Demographic, laboratory, and pregnancy outcome data were collected for 39 women whose antiretroviral treatment regimens were expanded to include more than one nucleoside analog for maternal indications. The $\mathbf{4 0}$ newborns were monitored at pediatric referral centers through at least three months of age to ascertain their HIV infection status.

Results: For all 39 women, zidovudine (ZDV) therapy was instituted at $13.4 \pm 8.2$ weeks, with a second agent (lamivudine [3TC] in $85 \%$ of cases) being added at a mean gestational age of 17.6 weeks. Duration of therapy with two agents was $20.6 \pm 10.4$ weeks overall, with no women stopping medications because of side effects or toxicity. No significant changes in maternal laboratory values were seen, except for an increase in mean corpuscular volume, over the course of pregnancy. No clinically significant adverse neonatal outcomes were noted, with all but the three preterm newborns leaving hospital with their mothers. Neonatal anemia (hematocrit $<50 \%$ ) was seen in $62 \%$ of newborns, with no children needing transfusion; mild elevations of liver function tests, primarily aspartate aminotransferase, were noted in $58 \%$ of newborns tested, though none were clinically jaundiced. Overall rate of neonatal HIV infection was 2.5\% (95\% confidence interval: 0.1-13.2\%).

Conclusion: Combination antiretroviral therapy during pregnancy with two nucleoside analogs was well-tolerated by mothers and newborns, with no significant short-term toxicities or side effects noted. Surveillance of exposed newborns' hematologic and liver function appears warranted. Infect. Dis. Obstet. Gynecol. 6:237-243, 1998. @ 1999 Wiley-Liss, Inc.
\end{abstract}

antiretrovirals; pregnancy; infectious complications; HIV infection; vertical transmission

*Correspondence to: Dr. Neil S. Silverman, Cedars-Sinai Medical Center, Department of Obstetrics and Gynecology, Division of Maternal-Fetal Medicine, 8700 Beverly Boulevard, Suite 160W, Los Angeles, CA 90046. E-mail: silvermann@eshs.org 
$\mathbf{R}_{\mathrm{f}}^{\mathrm{c}}$ ecent advances in the treatment of adults infected with the human immunodeficiency virus (HIV) have established combination antiretroviral therapy as the current standard for treatment of these individuals. The most recent guidelines proposed by both the US Department of Health and Human Services and the International AIDS Society reinforce this approach, ${ }^{1,2}$ emphasizing that treatment decisions include evaluation of an individual patient's immune status via HIV-RNA and CD4+ cell analyses, but stressing the use of at least two, and ideally three, antiretroviral medications at the initiation of therapy. The ultimate goal of all HIV therapeutic regimens is designed to be the suppression of HIV-RNA below detectable levels, which has been shown to be directly related to the risk of disease progression. ${ }^{3}$

With HIV infection, as with other medical conditions, a coexisting pregnancy presents unique concerns regarding appropriateness and safely of treatment options, even those that have clearly been of benefit for nonpregnant adults. A regimen of maternal and neonatal therapy with zidovudine (ZDV) in HIV-exposed pregnancies has been shown in a large multicenter trial to significantly decrease the risk of vertical HIV transmission by nearly $70 \%$, from $25 \%$ to $8 \% .^{4}$ This therapy was well-tolerated by the women participating in the trial, with neonatal effects appearing to be limited to mild transient anemia. ${ }^{4}$ Subsequent singlecenter or regional reports have demonstrated similarly encouraging results regarding interruption of vertical HIV transmission with the use of all or only some of the treatment components of the larger trial's ZDV protocol. ${ }^{5-8}$ These positive results have led the United States Public Health Service (USPHS) to recommend offering HIV counseling and testing to all pregnant women, so that women diagnosed as HIV-infected can be offered treatment that would both decrease the risk of neonatal infection and delay the progression of maternal disease. ${ }^{9,10}$

The current adult guidelines recognize that the trials to interrupt perinatal HIV transmission, using ZDV alone for antepartum/intrapartum/neonatal therapy, stand in contrast to evolving standards for adult HIV therapy. The most recent USPHS recommendations for the treatment of HIV-infected pregnant women, in fact, now state that "therapies of known benefit to women should not be withheld during pregnancy unless ....adverse effects outweigh the benefit to the woman," 11 an approach supported by other experts in the obstetric literature. ${ }^{12}$ However, the authors of these guidelines acknowledge a paucity of human neonatal sideeffect data regarding in utero exposure to antiretroviral agents other than $Z \mathrm{DV}$.

Data regarding the safety of antiretroviral agents other than ZDV during pregnancy, to date, are limited to abstract presentations or small series evaluating late-pregnancy treatment. ${ }^{13-15}$ Many clinicians providing care to $\mathrm{HIV}$-infected women during pregnancy have used medications other than ZDV for maternal indications, encouraged by any lack of association reported between congenital malformations and maternal treatment with other nucleoside analogs, specifically ZDV and acyclovir. ${ }^{4,16,17}$ Therefore, we sought to report on our centers' preliminary, cumulative experience with combination antiretroviral regimens for HIVinfected mothers, for whom treatment had been designed with the intent of maximal viralsuppressive benefit for both the women and their newborns. While our series is not intended to supplant larger registry reports, we felt it important to combine our early treatment experiences in light of the recommendations for expanded maternal therapy outlined in the new perinatal HIV treatment guidelines. ${ }^{11}$ While limited in size, we intended our series to begin to fill the gaps that currently exist in counseling pregnant patients in need of appropriately aggressive antiretroviral therapies.

\section{METHODS}

The study population consisted of all HIV-infected women referred for counseling and prenatal care at the referring centers. From 1995 through December 1997, women at these centers who were either newly diagnosed with HIV infection or known to be infected but previously untreated were offered combination antiretroviral therapy with zidovudine $(\mathrm{AZ} T)$ and an additional nucleoside analog. Each woman underwent individual counseling regarding pregnancy options and HIV treatment options. The results of the multicenter AZT trial (AIDS Clinical Trial Group Protocol \#076) ${ }^{4}$ were discussed in detail, and recommendations for the use of at least AZT to decrease perinatal HIV transmission were outlined. ${ }^{10}$ However, the clinicians at these centers also discussed the use of additional 
antiretroviral agents to favorably impact on maternal viral load and disease progression and possibly to augment the prophylactic impact on the newborn. The lack of neonatal exposure and safely data was discussed with all women in the context of risk-benefit decisions.

As the observation period progressed, all centers encountered women who had been diagnosed prior to the index pregnancies and had been on combination regimens preconceptionally. After discussions with these women, any successful HIV treatment regimens were continued in the absence of maternal disease progression.

At the beginning of the study period, women naïve to antiretroviral therapy with favorable lymphocyte subset profiles (CD4+ subset > 500 cells/ $\mathrm{mm}^{3}$ ) were placed on AZT alone in accordance with Centers for Disease Control and Prevention (CDC)/USPHS guidelines, ${ }^{10}$ with a second agent added if predetermined parameters (CD4+ subset or HIV-RNA levels) deteriorated. Not all centers used viral RNA levels uniformly throughout the observation period. As guidelines became established in nonpregnant adult populations, drugnaïve women meeting adult criteria for treatment outside of pregnancy were begun concomitantly on two agents at 12-14 weeks of gestation.

Hematologic, serum chemistry, and lymphocyte subset values were monitored through the women's pregnancies, with complete blood counts performed on all and liver function testing performed on most newborns. Complete nursery examinations were performed on all newborns by the centers' pediatric staffs, and delivery statistics were collected on all exposed newborns. Neonatal virologic status was sought on all exposed infants. All children were treated with oral $Z \mathrm{DDV}$ in accordance with established pediatric guidelines.

Continuous data were compared by both paired and unpaired Student t-tests, with noncontinuous paired data analyzed with Wilcoxon signed rank tests. Statistically significant differences between comparison groups were defined at a level of $P<$ 0.05 , with $95 \%$ confidence levels assigned where appropriate.

\section{RESULTS}

The investigators at the six contributing centers monitored $39 \mathrm{HIV}$-infected women, each of whom
TABLE I. Maternal antiretroviral therapy

\begin{tabular}{lcc}
\hline & $\begin{array}{c}\text { Mean } \\
\left( \pm S D^{a}\right)\end{array}$ & $\begin{array}{c}\text { Median } \\
(\text { range })\end{array}$ \\
\hline Gestational age ZDV started (weeks) & $13.4 \pm 8.2$ & $12(6-36)$ \\
Gestational age 2nd agent started & & \\
$\quad$ (weeks) & $17.6 \pm 10.7$ & $14(6-37)$ \\
$\quad$ If patient symptomatic $(n=5)$ & $8.0 \pm 4.0$ & \\
$\quad$ If patient asymptomatic $(n=34)$ & $18.6 \pm 10.3$ & \\
Duration of combined therapy (weeks) & $20.6 \pm 10.4$ & $22(3-34)$ \\
\hline
\end{tabular}

${ }^{\mathrm{a}} \mathrm{SD}$, standard deviation.

was treated with two nucleoside analogs during their pregnancies, and who delivered 40 live newborns (one set of twins) among them. These women, with a mean age of $27.4 \pm 5.2$ years, had been diagnosed with HIV infection for a median of two years (range: $0-11$ years), with 14 (35.9\%) diagnosed with the index pregnancy, and $29(74.4 \%)$ diagnosed for less than five years. Only five (13\%) had had symptomatic sequelae of their HIV infection; in two of these women, pneumocystis carinii pneumonia had been the presenting illness that prompted diagnosis prior to pregnancy. The majority of women $(69 \%)$ identified heterosexual contact alone as their infection risk, with $28 \%$ reporting prior or ongoing drug use, and one woman (3\%) having acquired her infection through past transfusion of blood products.

For all women, ZDV therapy $(200 \mathrm{mg}$, orally, three times a day) was instituted at 12-14 weeks of gestation (or at the first visit, if later) if antiretroviral treatment was not continuing from the prepregnancy period. As seen in Table 1, ZDV was begun soon after completion of the first trimester in the majority of women; only five women were placed on ZDV after 20 weeks of gestation, all of whom presented late for care and were diagnosed as HIV-infected during the index pregnancy.

Combination nucleoside analog therapy was instituted later in pregnancy on average, though as the study period progressed, more women either entered pregnancy already on two agents or were concurrently placed on two agents initially at the prenatal center. Overall, 12 women $(30.8 \%)$ were already on two nucleoside analogs at the time of presenting for prenatal care, $13(33.3 \%)$ were placed on two agents upon entry to care, with the remaining 14 (35.9\%) placed on a second agent some time in the second or third trimester, either for progressive decreases in $\mathrm{CD} 4+$ cell counts or 
TABLE 2. Maternal laboratory values $(n=39)^{a}$

\begin{tabular}{lcc}
\hline & At entry & At delivery \\
\hline WBC $\left(10^{3} / \mathrm{L}\right)$ & $5.8 \pm 1.6$ & $7.5 \pm 2.6^{*}$ \\
Hemoglobin $(\mathrm{g} / \mathrm{dL})$ & $11.5 \pm \mathrm{I} . \mathrm{I}$ & $11.4 \pm 1.5$ \\
$\mathrm{MCV}(\mathrm{fL})$ & $93 \pm 10.5$ & $102.3 \pm 11.5^{*}$ \\
Platelets $\left(\mathrm{I} 0^{3} / \mathrm{L}\right)$ & $220 \pm 61$ & $220 \pm 58$ \\
AST $(\mathrm{IU} / \mathrm{L})$ & $26.8 \pm 12.7$ & $28.7 \pm 13.7$ \\
ALT $(\mathrm{IU} / \mathrm{L})$ & $23.7 \pm 15.7$ & $23.9 \pm 17.3$ \\
CD4 (median cells/ & & \\
$\mathrm{mm}^{3}$, range) & $274(30-932)$ & $340(45-81 \mathrm{I})^{* * *}$ \\
\hline
\end{tabular}

${ }^{\mathrm{a}} \mathrm{All}$ means \pm standard deviation except where indicated.

$* P<0.00$ I by paired t-tests.

$* * P=0.06$ by Wilcoxon signed rank test.

increases in HIV-RNA levels, depending on the center. The majority of women were given lamivudine (3TC) as the second nucleoside analog (33/39; $84.6 \%$ ), with four women treated with didanosine (ddI), and two with zalcitabine (ddC). For the purposes of analysis, the presence of a second nucleoside analog during the pregnancy was not stratified by agent used, due to the small size of the ddI and ddC groups.

Table 2 shows the maternal laboratory values followed during the women's pregnancies. There were increases noted in the white blood cell (WBC) counts and the mean corpuscular volumes (MCV) in women when paired analyses comparing entry values to delivery values were performed. No significant increases were noted in other hematologic values, liver function tests, or median CD4+ cell counts. No women discontinued their therapy during pregnancy as a result of either laboratory toxicity or unacceptable side effects.

Table 3 describes delivery data for the 40 newborns. As shown, the majority of pregnancies were delivered at 36 weeks of gestation or more. The three women who delivered at earlier gestational ages (at 32, 33, and 35 weeks) did so for non-HIVrelated complications (one for chronic hypertension and fetal distress, and two for refractory preterm labor without clinical chorioamnionitis). Nearly three fourths of the women delivered vaginally (71.8\%; 95\% confidence interval $[\mathrm{CI}]=55.1-85 \%)$; the 11 women who delivered via cesarean delivery had surgery performed for obstetric indications (breech presentation, arrest of dilatation, and active genital herpes) rather than maternal HIV-related concerns.

Neonatal outcome data from the 39 pregnancies are summarized in Table 4 . No clinical dysmor-
TABLE 3. Delivery data

\begin{tabular}{lc}
\hline Gestational age at delivery (weeks) & $38.2 \pm 2.1$ \\
& $($ median $=38 \mathrm{wk}$ ) \\
Delivery $\geq 36$ weeks & $36(92.3 \%)$ \\
Birthweight (grams) & $2979 \pm 667$ \\
Birthweight for all $\geq 36$ weeks $(\mathrm{n}=37)$ & $3068 \pm 597$ \\
Vaginal delivery & $28(71.8 \%)$ \\
\hline
\end{tabular}

TABLE 4. Newborn laboratory values $(n=40)$

\begin{tabular}{lc}
\hline WBC $\left(I 0^{3} / \mathrm{L}\right)$ & $13.2 \pm 4.7$ \\
Hemoglobin $(\mathrm{g} / \mathrm{dL})$ & $15.6 \pm 3.0$ \\
Hematocrit $(\%)$ & $47 \pm 8.9$ \\
Platelets $\left(\mathrm{I} 0^{3} / \mathrm{L}\right)$ & $266 \pm 97$ \\
AST $(\mathrm{IU} / \mathrm{L})[\mathrm{n}=24]$ & $46 \pm 20$ \\
ALT $(\mathrm{IU} / \mathrm{L})[\mathrm{n}=24]$ & $15 \pm 10$ \\
Anemia $(\mathrm{Hct}<50 \%)$ & $25(62.5 \%)$ \\
$\quad$ Mean hematocrit for anemic newborns (\%) & $41.2 \pm 5.5$ \\
$\quad$ Mean hematocrit for nonanemic newborns $(\%)$ & $55.2 \pm 6.2$ \\
Elevated ALT or AST (>35 IU/L) & $14 / 24(58.3 \%)$ \\
$\quad$ ALT or AST >I.5 $\times$ upper limit & \\
$\quad$ of normal range & $6 / 24(25 \%)$ \\
\hline
\end{tabular}

phology was identified in any of these children, and, despite a rate of neonatal anemia (defined as hematocrit $<50 \%)$ of $62 \%(95 \% \mathrm{CI}=45.8-77.3 \%)$, none of the newborns required blood transfusion. No clinically significant laboratory abnormalities were identified in these children, though it is notable that of the 24 newborns with liver function tests available, $14(58.3 \%)$ had elevations of either alanine aminotransferase (ALT) or aspartate aminotransferase (AST), defined as a value higher than $35 \mathrm{IU} / \mathrm{L}$. When elevated neonatal liver function tests were defined as ALT or AST greater than 1.5 times the upper limit of normal, however, only six of 24 newborns $(25 \%)$ met those criteria. None of these children were clinically jaundiced or required phototherapy, however, and all but the three preterm newborns were discharged home with their mothers.

The nature of this report precludes conclusions about the effectiveness of double-nucleoside therapy during pregnancy for interrupting vertical HIV transmission, and maternal HIV-RNA levels were not followed consistently enough among the centers to remark on trends or effects. However, virologic status through at least three months of age is available on all the newborns in this study, with only one diagnosed as HIV-infected by both polymerase chain reaction testing of HIV-RNA and by viral culture, giving a vertical transmission rate in this series of $2.5 \%(95 \% \mathrm{CI}=0.1-13.2 \%)$. Of note 
is the fact that this child's mother had a documented acute HIV seroconversion during the index pregnancy.

\section{COMMENTS}

Recent guidelines for the treatment of HIVinfected pregnant women have brought antiretroviral recommendations for this population into concordance with those for other adult and adolescent populations. ${ }^{2,11}$ Treatment regimens, therefore, should be designed to maximize benefit for the mother, with the presumption that optimal maternal viral suppression will also contribute to lowering the risk of vertical HIV transmission. ${ }^{18-21}$

The integration of ZDV therapy into antepartum and intrapartum management of HIV-infected women has contributed significantly to the documented decline in the incidence of perinatallyacquired HIV infection in the United States. ${ }^{22} \mathrm{Be}-$ cause perinatal transmission of HIV now accounts for virtually all new HIV infections in children, ${ }^{23}$ maximizing the effectiveness of therapies designed to interrupt vertical transmission is of critical importance.

Through collaborative compilation of data from six perinatal centers, we have been able to present a preliminary experience with the use of combination nucleoside analog therapy during pregnancy. These outcomes predate the inclusion of protease inhibitors in most adult combination regimens and reflect the intent of the investigators to augment maternal therapy by using additional medications from a class for which neonatal exposure and safety profiles had at least been previously described. ${ }^{4,16,17}$ For the most part, lamivudine was the second agent of choice, since its acceptability to patients in terms of dosing and side effects had been well-described in other adult groups. ${ }^{24,25}$ In addition, the transplacental passage of lamivudine in laboratory models using human placentas has been shown to result in favorable maternal-fetal compartment ratios. ${ }^{26}$ All investigators maintained ZDV therapy during the women's pregnancies, since it is the only agent for which interruption of vertical HIV transmission has been documented, ${ }^{4}$ though it was expected that other similar agents in combination would have at least comparable if not improved effects on these transmission rates.

Our results demonstrate that combination nucleoside therapy was well-tolerated by all the women, with none of them discontinuing treatment for side effects or laboratory toxicities. Nearly all of the women delivered at term, similar to those participating in the ACTG-076 trial, ${ }^{4}$ with maternal HIV status in our series not related to route of delivery. There were no clinically significant differences in laboratory values monitored in these women from the time of entry into care through delivery. While HIV-RNA levels would have been interesting to monitor, they were not drawn consistently enough across the centers to allow for appropriate data analysis.

These initial data also indicate that combination nucleoside therapy appeared to be well-tolerated by the exposed children. Rates of neonatal anemia were comparable to those in populations of children exposed to $\mathrm{ZDV}$ alone, ${ }^{4}$ with no transfusions required; all other hematologic parameters were within normal neonatal ranges. It is notable that elevations of liver function tests were seen in children exposed to combination therapy, an effect not observed in the larger multicenter ZDV-only trial. ${ }^{4}$ Not all of the children in our series were monitored longitudinally, though none were clinically jaundiced at birth, and it appeared to be the serum AST that was elevated selectively, with a mean value approximately $25 \%$ above the upper normal limit of $35 \mathrm{IU} / \mathrm{L}$ for this test. In addition, if significant transaminase elevations are considered to be those greater than 1.5 times the upper limit of normal, only $25 \%$ of exposed newborns would have met those criteria.

The small sample size of this series precludes substantial comment on the value of combination therapy to augment the impact of $\mathrm{ZDV}$ alone in interrupting vertical HIV transmission. ${ }^{4}$ In contrast to the initial ZDV treatment trial, women in this series had lower median CD4+ cell counts at entry and were not excluded from therapy for symptomatic status. Still, our overall rate of neonatal HIV infection was only $2.5 \%$, which is at least comparable to the 5-8\% rates reported in other randomized and nonrandomized series of treatment with ZDV alone. ${ }^{4,8}$ Definitive conclusions about the impact of combination therapy on vertical transmission of HIV, however, are beyond the scope of this preliminary report.

Our combined experience does provide early objective support for the use of combination nucleoside analog therapy for the treatment of 
HIV-infected women during pregnancy when regimens are intended to maximize antiretroviral benefit for the women. An additional side-benefit in further decreasing the risk of vertical HIV transmission beyond that demonstrated for ZDV alone may ultimately be proven. However, this series does describe the first maternal and neonatal safety profiles for combination HIV therapy administered through the major portion of pregnancy in HIVinfected women in accordance with recently revised CDC guidelines. ${ }^{11}$ Hematologic and liver function profiles in exposed newborns appear to warrant careful surveillance, though no significant short-term neonatal morbidities or toxicities were identified. Even as the nature of treatment of chronic HIV-related illness continues to progress, these early results should greatly assist in the counseling and medical management of HIV-infected pregnant women until larger registry reports become available.

\section{REFERENCES}

1. Guidelines for the use of antiretroviral agents in HIVinfected adults and adolescents. MMWR Morb Mortal Wkly Rep 47 (RR-5):43-82, 1998.

2. Carpenter CJ, Fischl MA, Hammer SM, et al.: Antiretroviral therapy for HIV infection in 1997. Updated recommendations of the International AIDS SocietyUSA Panel. JAMA 277:1962-1969, 1997.

3. Mellors JW, Munoz A, Giorgi JV, et al.: Plasma viral load and CD4+ lymphocytes as prognostic markers of HIV-1 infection. Ann Intern Med 126:946-954, 1997.

4. Connor EM, Sperling RS, Gelber R, et al.: Reduction of maternal-infant transmission of human immunodeficiency virus type 1 with zidovudine treatment. N Engl J Med 331:1173-1180, 1994.

5. Simpson BJ, Shapiro ED, Andiman WA: Reduction in the risk of vertical transmission of HIV-1 associated with treatment of pregnant women with orally administered zidovudine alone. J Acquir Immune Defic Syndr Hum Retrovirol 14:145-152, 1997.

6. Fiscus SA, Adimora AA, Schoenbach VJ, et al.: Perinatal HIV infection and the effect of zidovudine therapy on transmission in rural and urban counties. JAMA 275: 1483-1488, 1996.

7. Matheson PB, Abrams EJ, Thomas PA, et al.: Efficacy of antenatal zidovudine in reducing perinatal transmission of human immunodeficiency virus type 1 . J Infect Dis 172:353-358, 1995.

8. Boyer PJ, Dillon M, Navai M, et al.: Factors predictive of maternal-fetal transmission of HIV-1. Preliminary analysis of zidovudine given during pregnancy and/or delivery. JAMA 271:1925-1930, 1994.

9. Centers for Disease Control and Prevention: U.S. Public Health Service recommendations for human immuno- deficiency virus counseling and voluntary testing for pregnant women. MMWR Morb Mortal Wkly Rep 44(RR-7):1-14, 1995.

10. Centers for Disease Control and Prevention: Recommendations of the Public Health Service Task Force on use of zidovudine to reduce perinatal transmission of human immunodeficiency virus. MMWR Morb Mortal Wkly Rep 43 (RR-11):1-21, 1994.

11. Centers for Disease Control and Prevention: Public Health Service Task Force recommendations for the use of antiretroviral drugs in pregnant women infected with HIV-1 for maternal health and for reducing perinatal HIV-1 transmission in the United States. MMWR Morb Mortal Wkly Rep 47(RR-2):1-30, 1998.

12. Minkoff $\mathrm{H}$, Augenbraun M: Antiretroviral therapy for pregnant women. Am J Obstet Gynecol 174:478-489, 1997.

13. Kleinman GE, Maher JE, Navarro D, et al.: The effects of combination retroviral therapy on the viral load and CD4 count in a regional obstetric HIV clinic. Proceedings of the 18th Annual Meeting of the Society of Perinatal Obstetricians, Miami, FL, February 2-7, 1998. Am J Obstet Gynecol 178 (part 2):S210, 1998.

14. Moodley J, Moodley D, Pillay K, et al.: Antiviral effect of lamivudine alone and in combination with zidovudine in HIV-infected pregnant women. Proceedings of the Fourth Conference on Retroviruses and Opportunistic Infections, Washington, DC, January 22-26, Abstract 607, p. 176, 1997.

15. Johnson MA, Goodwin C, Yuen GJ, et al.: The pharmacokinetics of 3'TC administered to HIV-1 infected women (pre-partum, during labour and post-partum) and their offspring. XIth International Conference on AIDS, Vancouver BC, July 7-12, 1996. Vol I, Abstract Tu.C.445, pp 249-250, 1996.

16. Kumar RM, Hughes PF, Khurranna A: Zidovudine use in pregnancy: a report on 104 cases and the occurrence of birth defects. J Acquir Immune Defic Syndr 7:1034 1039, 1994.

17. Centers for Disease Control and Prevention: Pregnancy outcomes following systemic prenatal acyclovir exposure-June 1, 1984-June 30, 1993. MMWR Morb Mortal Wkly Rep 42:806-809, 1993.

18. Cao Y, Krogstad P, Korber B'T, et al.: Maternal HIV-1 viral load and vertical transmission of infection: the Ariel Project for the prevention of HIV transmission from mother to infant. Nature Med 3:549-552, 1997.

19. Mayaux MJ, Dussaix E, Isopet J: Maternal virus load during pregnancy and mother-to-child transmission of human immunodeficiency virus type 1: the French perinatal cohort studies. J Infect Dis 175:172-175, 1997.

20. Coll O, Hernandez M, Boucher CAB, et al.: Vertical HIV-1 transmission correlates with a high maternal viral load at delivery. J Acquir Immune Defic Syndr Hum Retrovirol 14:26-30, 1997.

21. Sperling RS, Shapiro DE, Coombs RW, et al.: Maternal viral load, zidovudine treatment, and the risk of transmission of human immunodeficiency virus type 1 from mother to infant. N Engl J Med 335:1621-1629, 1996. 
22. Centers for Disease Control and Prevention: Update: perinatally acquired HIV/AIDS-United States, 1997. MMWR Morb Mortal Wkly Rep 46:2-8, 1997.

23. Centers for Disease Control and Prevention: HIV/AIDS Surveillance Report. Atlanta, GA: U.S. Department of Health and Human Services, Public Health Service, 1996 (Vol 8, no. 2).

24. Eron JJ Jr.: The treatment of antiretroviral-naïve subjects with the $3 \mathrm{TC} /$ zidovudine combination a review of
North American (NUCA 3001) and European (NUCB 3001) trials. AIDS 10:S11-19, 1996.

25. Perry CM, Faulds D: Lamivudine: a review of its antiviral activity, pharmacokinetic properties and therapeutic efficacy in the management of HIV infection. Drugs 53:657-680, 1997.

26. Bloom SL, Dias KM, Bawdon RE, Gilstrap LC: The maternal-fetal transfer of lamivudine in the ex vivo human placenta. Am J Obstet Gynecol 176:291-293, 1997. 


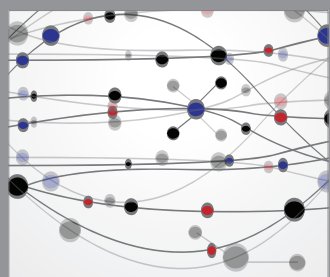

The Scientific World Journal
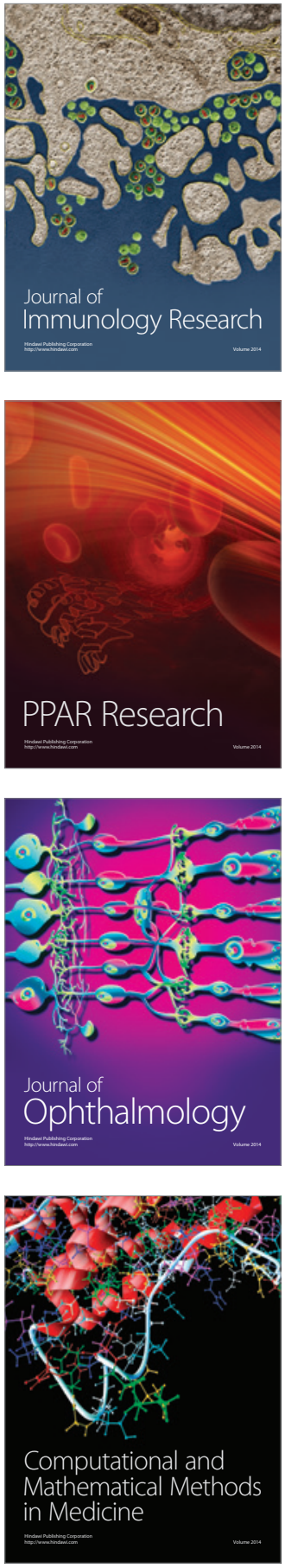

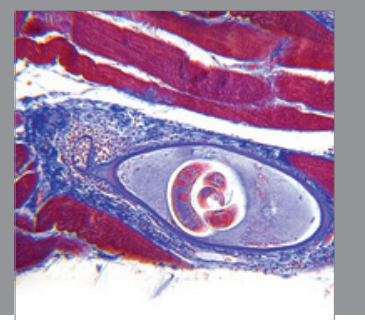

Gastroenterology

Research and Practice
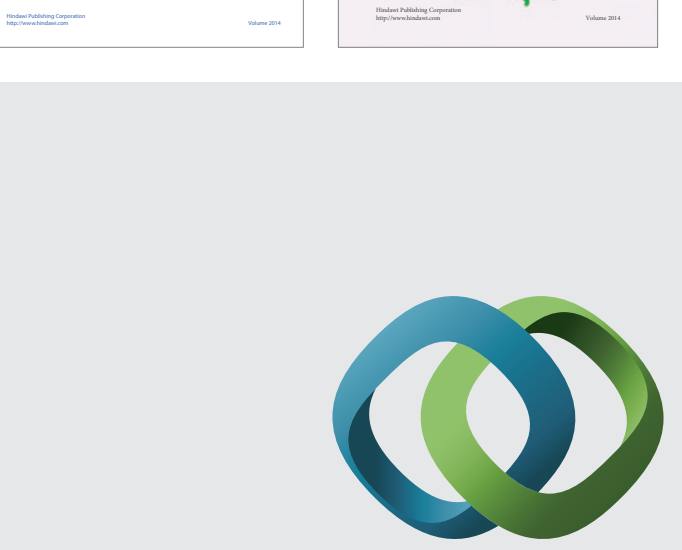

\section{Hindawi}

Submit your manuscripts at

http://www.hindawi.com
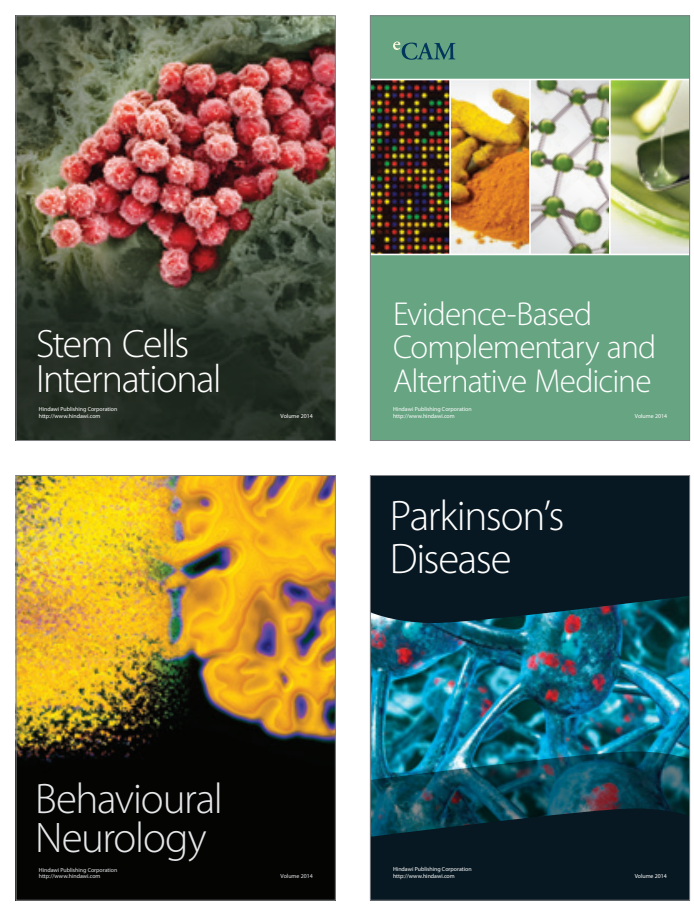

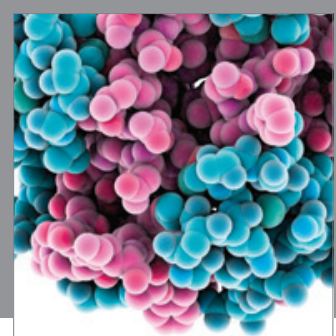

Journal of
Diabetes Research

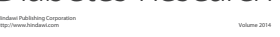

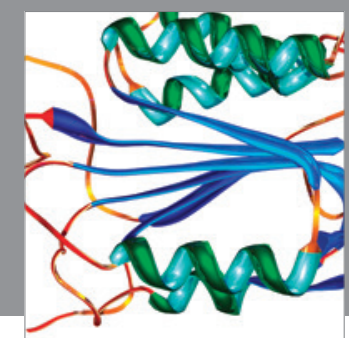

Disease Markers
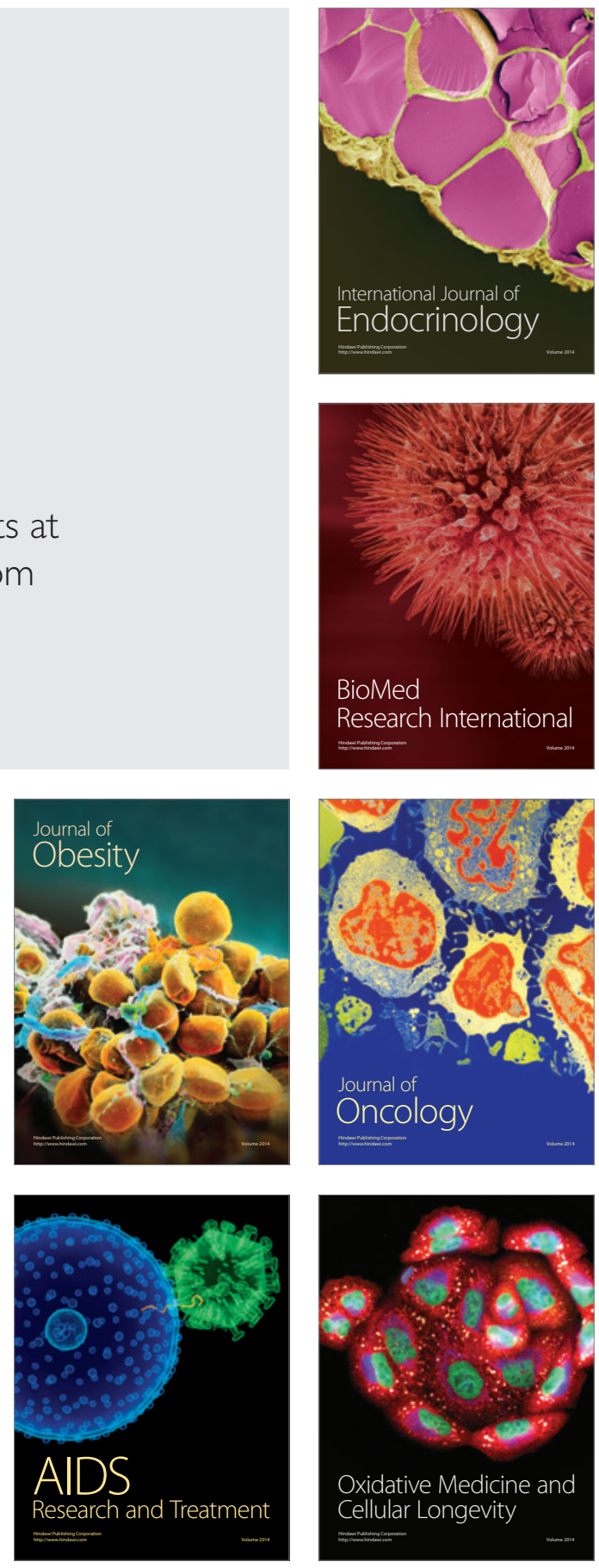